\title{
Comparative Study Of Photodynamic Therapy With Riboflavin-Tryptophan Gel And 13\% 5-Aminolevulinic Acid In The Treatment Of Mild To Moderate Acne Vulgaris
}

This article was published in the following Dove Press journal:

Clinical, Cosmetic and Investigational Dermatology

\section{Suparat Wangsuwan Jitlada Meephansan (D)}

Division of Dermatology, Chulabhorn International College of Medicine, Thammasat University, Pathum Thani 12120, Thailand
Correspondence: Jitlada Meephansan Division of Dermatology, Chulabhorn International College of Medicine,

Thammasat University, Rangsit Campus, Klong Luang, Pathum Thani 12120 , Thailand

Tel +66 025644444 Ext 4495

Email kae_mdcu@yahoo.com
Background: Non-invasive photodynamic therapy (PDT) with 5-aminolevulinic acid (ALA) has been used as an alternative acne treatment for many years. Riboflavin and tryptophan are newly introduced photosensitizers.

Objective: To compare the efficacy of PDT with riboflavin-tryptophan (RT) gel with that of $13 \%$ ALA for the treatment of facial acne.

Methods: We performed a double-blind, split-face study of 37 subjects with mild to moderate facial acne vulgaris. RT gel was applied to half of the face, and 13\% ALA was applied to the other half. Then, the whole face was irradiated using a blue light-emitting diode for 20 mins. Four treatment sessions were performed over a 4-week duration, at 1week interval. The acne lesion counts and acne severity were assessed. Sebum secretion, Propionibacterium acne colonization, pore size, and skin texture were also evaluated.

Results: PDT with RT gel demonstrated good efficacy, non-inferior to ALA for acne treatment, with a significant reduction in acne lesion counts, severity grading, porphyrin, and sebum output. The pore size and skin texture were improved. Side-effects were minimal and well tolerated in all subjects.

Conclusion: PDT therapy with RT gel is an effective alternative treatment for acne vulgaris. Keywords: acne vulgaris, photodynamic therapy, 5-aminolevulinic acid, riboflavin, tryptophan

\section{Introduction}

Acne vulgaris is a common inflammatory disease and affects all age groups, with a higher prevalence in adolescents and young adults. The four main antecedents for acne maturation are follicular hyperkeratinization, Propionibacterium acnes colonization, sebum production, and inflammatory processes. ${ }^{1}$ The first-line treatments for mild to moderate acne are benzoyl peroxide, topical retinoids, or a topical therapy-antibiotic combination. ${ }^{1}$ However, these orthodox treatments can cause bacterial resistance, irritation, and systemic side-effects. ${ }^{1,2}$

Photodynamic therapy (PDT) has been used as an alternative treatment because of advantages including better compliance and a lower risk of bacterial resistance. ${ }^{3,4}$ PDT combines a photosensitizer and activating light to create a photochemical reaction ${ }^{5}$ and it is a non-invasive modality. However, the efficacy of PDT for the treatment of acne vulgaris remains unclear. ${ }^{6}$ PDT promotes acne improvement by using both a short 
application time with a blue light or a long application time under occlusion with high red light doses. ${ }^{3}$ PDT reduces acne through direct photodynamic effects on sebaceous glands, ${ }^{7}$ diminution of follicular obstruction and hyperkeratosis, ${ }^{8}$ and immunological alterations. ${ }^{9,10}$

5-aminolevulinic acid (ALA) and methyl aminolevulinate (MAL) are the most widely used photosensitizers for acne treatment. PDT with ALA has been suggested for the downregulation of infiltrating inflammatory cells inside acne lesions. $^{3}$ ALA-mediated photodynamic reactions directly destroy $P$. acnes and damage the pilosebaceous glands, thus leading to a clinical improvement in acne lesions. ${ }^{11}$ However, pain, adverse inflammatory effects, and residual photosensitivity are usually experienced during PDT treatment with topical ALA. ${ }^{12}$ Additionally, PDT with ALA can cause acute erythema and edema, blistering, oozing, and exfoliation, which could lead to hyperpigmentation ${ }^{7}$ and may cause drug interactions with griseofulvin, thiazines, sulfonylureas, sulfonamides, cyclophosphamide, or tetracyclines. ${ }^{13,14}$

Riboflavin (vitamin B2) has been introduced as a natural, potent photosensitizing molecule. ${ }^{15}$ Riboflavin is important for the conversion of tryptophan to niacin. ${ }^{16}$ Riboflavin has a peak absorption around 365-445 nm. After visible light irradiation, especially blue light, this micronutrient can generate singlet-excited states. ${ }^{15}$ They are safe, natural substances without any reported sideeffects.

The aim of this study was to evaluate the efficacy and safety of PDT using a riboflavin-tryptophan (RT) gel similar to that of $13 \%$ ALA for the treatment of mild to moderate facial acne vulgaris.

\section{Methods}

\section{Subjects}

This was a single-center, double-blinded split-face comparative study. The study was approved by the Human Research Ethics Committee of the Thammasat University No.1, conducted according to the principles of the Declaration of Helsinki, and registered in the Thai Clinical Trials Registry (No. TCTR20190907005). Patients provided written informed consent before study initiation and accompanying photographic images to be published. Individual participant data of the study, after deidentification, are not being shared with others. We do not share any specific data, and no other study-related documents will be made available. The case record form and essential documents will be kept in a designated place for 1 year. The data and documents are available if requested by relevant authorities.

Thirty-seven patients aged $18-50$ years presenting to the Out Patients Department (OPD) of Dermatology, Benchakitti Park Hospital, who had been diagnosed with mild to moderate facial acne vulgaris. The exclusion criteria included a history of topical antibiotics and retinoids use for acne treatment within the previous 4 weeks, medication use that could aggravate or suppress acne (such as halogens, antiepileptics, antidepressants, ciclosporin, and vitamin B), photosensitizing drug use, photosensitivity, keloids, any other skin diseases, any other systemic diseases, oral contraceptive pill, and oral antibiotics use within the previous 3 months. History of PDT, laser or other procedural treatments use within previous 6 months. Pregnant and lactating women were also excluded. No other treatments for acne were allowed during the study.

\section{Materials}

There were two photosensitizers used in this procedure: A riboflavin-tryptophan (RT) gel from BR PDT Gel ${ }^{\circledR}$ in which the ingredients are riboflavin, tryptophan, glycolic acid, and niacinamide (BR PHARM Co. Ltd., Gangwondo, Korea); and 5-aminolevulinic acid (ALA) from a topical ALA (13\%)-mask Omega light (N. Finders Co., Ltd., Seoul, Korea). The light source came from an Omnilux PDT $^{\mathrm{TM}}$ LED System (GlobalMed Technologies Co., CA, USA).

\section{Treatment}

Patients underwent four full-face PDT treatments with a RT gel on one facial side and a 13\% topical ALA mask on the other side. The application side for the two photosensitizers was randomized before the study. The patients and primary investigator were blinded to the photosensitizers. Approximately $0.1 \mathrm{~mL}$ RT gel along with a half-plain facial mask sheet (no additive substance) and a half $13 \%$ topical ALA face mask were applied and covered under plastic film occlusion for 30 mins. After the incubation, the remaining products were cleaned off using normal saline. Then, the whole face was illuminated simultaneously with blue light (Omnilux PDT $^{\mathrm{TM}}$ LED System) at $415 \mathrm{~nm}, 48 \mathrm{~J} / \mathrm{cm}^{2}$, for 20 mins. The light source was maintained at a $5-\mathrm{cm}$ distance from the tip of the nose. After the irradiation, a wet dressing of gauze soaked in cooled normal saline was provided for 10 mins. Subjects were treated at 1-week interval for four sessions. 


\section{Assessment}

Clinical evaluations were performed by acne lesion counts, the Global Acne Assessment Score (GAAS). ${ }^{17}$ The severity of acne, based on the Global Acne Assessment Score (GAAS), was evaluated using a 5-point scale as follows: 0 , clear; 1, almost clear; 2, mild; 3, moderate; 4 , severe; and 5 , very severe. Success according to the GAAS on the 5point static scale was defined as a rating of 0 (clear) or 1 (almost clear).

Fluorescence photography was used to visualize $P$. acne populations using wood's lamp. The number of punctured red fluorescent dots in each tested area was blindly counted. The tested area was graded on the basis of the porphyrin coverage area: 1 : less than $25 \%, 2: 25 \%-50 \%$, 3: $50 \%-75 \%$, and 4 : more than $75 \%$. Sebum output was collected from both cheeks using a Sebumeter (SM815, Courage1Khazaka Electronic GmbH, Germany). We used Antera $3 \mathrm{D}^{\mathrm{TM}}$ (Miravex Ltd., Dublin, Ireland) to measure the pore size and texture of the facial skin. Side effects, including itching, pain, erythema, hyperpigmentation, and exfoliation were recorded during the treatment period. Clinical photographs were taken prior to and following the treatment, and at every follow-up (every 1 week during treatment and every 2 weeks after treatment) for 7 weeks.

\section{Outcome}

The primary efficacy end-points were the proportion of patients who achieved success based on the GAAS and mean reduction in acne lesion counts at week 3 from baseline using non-inferiority comparison.

The secondary outcomes were the proportion of patients who achieved success based on the GAAS and mean reduction in acne lesion counts at weeks 5 and 7 from baseline and the side effects of the two treatments. Differences in the pore size and texture of the facial skin between the treatments were considered.

\section{Statistical Analysis}

For the primary outcome, we set the non-inferiority margin assuming that the lower bound of the $95 \%$ CI of the proportion difference in success rate between the PDTALA and PDT-RT groups was not $<30 \%$. With a power of $80 \%$ and one-sided significance level of 0.05 , the sample size needed was determined to be 37 participants after adjusting for a dropout rate of $10 \%$.

Data were analyzed using IBM SPSS for Windows, version 22 (IBM Corp., Armonk, NY, USA). Statistical significance was defined as a $p$-value $<0.05$. Non-parametric analysis of covariance, using the Wilcoxon signed rank test, assessed the changes between (p-value) and within the two groups (p-value) for each parameter. The incidence of success based on the GAAS of 0 or 1 was analyzed using the McNemar test.

\section{Results}

Thirty-four subjects both males $(\mathrm{n}=9,25.6 \%)$ and females $(\mathrm{n}=25,71.4 \%)$, had completed the treatment and followups for this study. The mean age of the subjects was 28.26 \pm 8.4 years. There was no significant difference in acne grading between the two facial sides at the pre-treatment visit ( $\mathrm{p}$-value $=0.705)$.

The achieved treatment success rates at weeks 3, 5, and 7 in terms of the investigator's static global assessment (GAAS of clear or almost clear) were not significantly different between the two facial sides ( $p>0.05$; Figures $1 \mathrm{~A}$ and $2 \mathrm{~A}-\mathrm{D})$.

For the acne lesion counts, the mean of comedone acne showed a significant decrease from the baseline after week 1 of treatment in both facial sides. However, comedone acne showed a more significant improvement with PDT with RT gel than with PDT with ALA at the third week (p-value $=0.021)$. At the last follow-up, PDT with RT gel produced a significant decrease in comedone acne from 21.67 to 14.39 (p-value $<0.001$ ), whereas the PDT on the ALA side yielded significant decrease from 23.14 to 15.07 (p-value $<0.001$ ) (Figure 1B).

There was no difference in papule acne between PDT with RT gel and PDT with ALA in any follow-up sessions (p-value $>0.05$ ). The papule acne on the PDT with ALA facial side significantly improved starting from the first week to the last follow-up. Nonetheless, the difference between pre- and post-treatment in PDT with RT gel was not statistically significant (Figure 1C).

The porphyrin reduction in PDT with RT gel and PDT with ALA was not significantly different ( $\mathrm{p}$-value $>0.05$ ) (Figure $3 \mathrm{~A}$ and $\mathrm{B}$ ). Within the therapy groups, significant porphyrin reduction began at the second week ( $p$-value $=0.008)$ after the PDT with RT gel treatment, while the reduction started at the first week ( $\mathrm{p}$-value $=0.031)$ for PDT with ALA. At the last followup, there was a significant porphyrin reduction in PDT with RT gel and PDT with ALA from 1.9 to 1.56 (p-value <0.001) and from 1.93 to 1.64 (p-value $<0.001$ ), respectively (Figure 4A).

The difference in the baseline sebum level between the two facial sides was significant $(p$-value $=0.024)$. However, there 

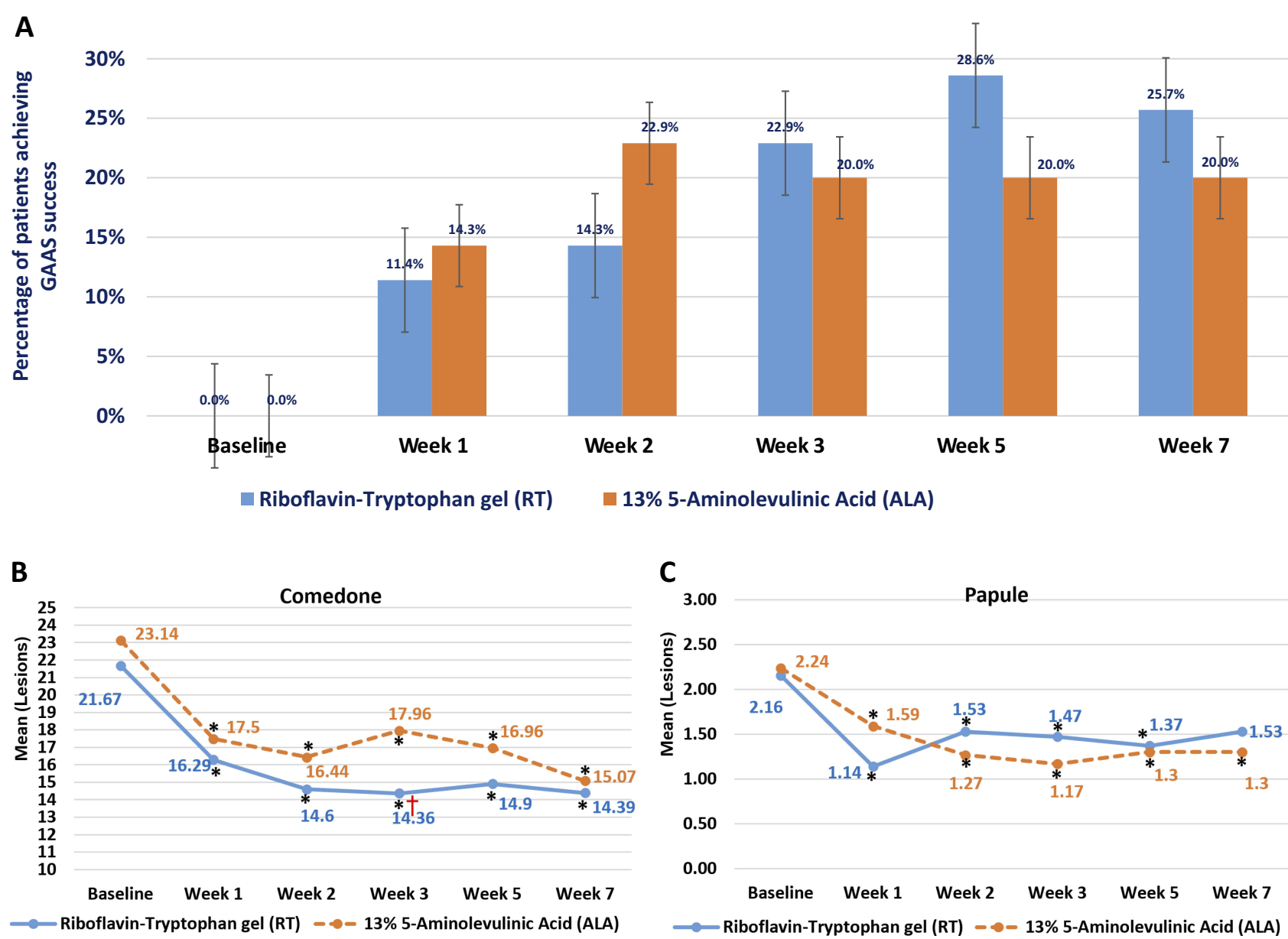

Figure I (A) Percentage of patients who achieved success based on the global acne assessment score of 0 (clear) or I (almost clear). (B) Changes in acne lesion counts; mean of comedones throughout the follow-up sessions. (C) Changes in acne lesion counts; mean of papules throughout the follow-up sessions. (*p-value $<0.05$ comparison to baseline within the group, ${ }^{\dagger} \mathrm{p}$-value $<0.05$ for comparison of PDT with RT gel and PDT with ALA).

A

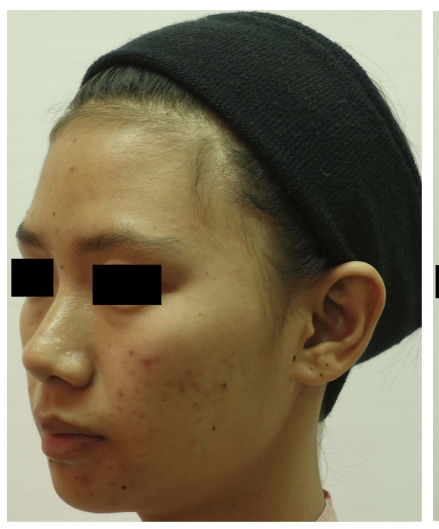

B

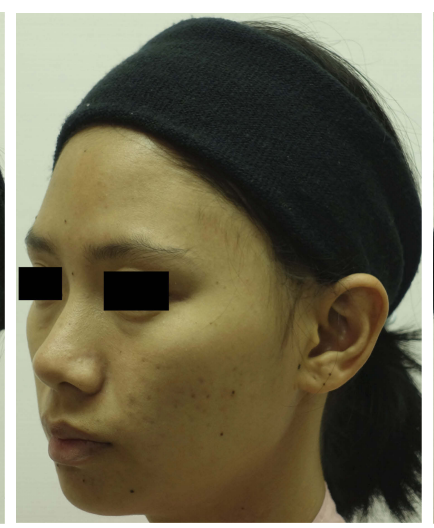

C

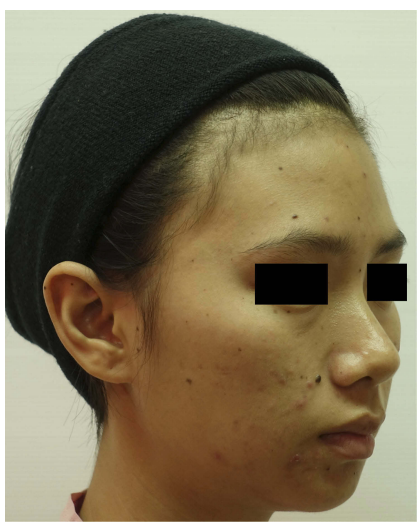

D

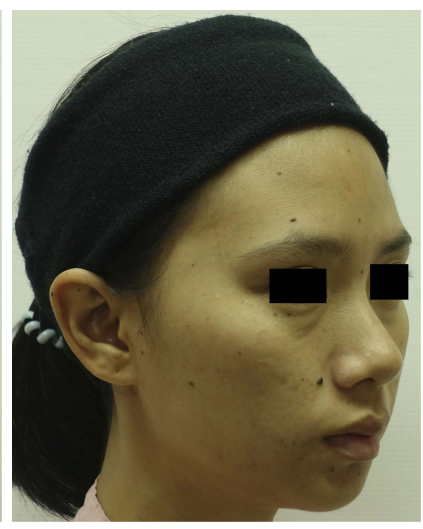

Figure 2 (A) Acne reduction compared between before (baseline) and (B) after treatment (at week 7) on the facial side treated with PDT with RT gel. (C) Acne reduction compared between before (baseline) and (D) after treatment (at week 7) on the facial side treated with PDT with ALA.

was no significant difference between the two facial sides from week 1 to 7 ( $p$-value $>0.05$ ). At the last follow-up, the mean sebum level showed a significant reduction from 17.53 at baseline to 7.97 (p-value $<0.001$ ) on the PDT with RT gel facial side and from 15.98 to 6.93 (p-value $<0.001$ ) in the PDT with ALA facial side (Figure 4B).

Examples of general appearance of the cheek are shown in Figures $5 \mathrm{~A}$ and $6 \mathrm{~A}$. Skin pore size was calculated as volume 

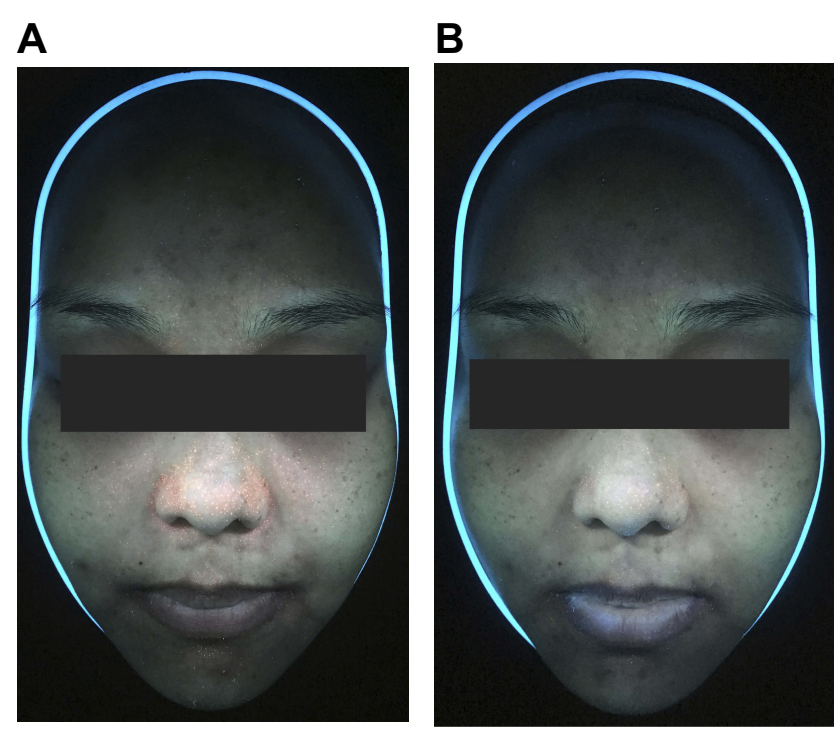

Figure 3 Fluorescence photographs $(\mathbf{A})$ before and $(\mathbf{B})$ after the treatment. In this patient, the right side of the face was treated with PDT with RT gel and the other side was treated with PDT with ALA.

$\left(\mathrm{mm}^{3}\right)$ as shown in Figures 5B and 6B. There was no statistically significant difference in the volume, which represented the skin pore size, between the two treatments ( $\mathrm{p}$-value $>0.05$ ). The facial pore size gradually decreased in both groups as seen in Figure 7. The median decrease in volume was from $3.4 \mathrm{~mm}^{3}$ to $2.38 \mathrm{~mm}^{3}$ in the PDT-RT gel group and from $3.4 \mathrm{~mm}^{3}$ to $2.8 \mathrm{~mm}^{3}$ in the PDT-ALA group. The skin texture was analyzed by the roughness index (Figures 5C and 6C). The skin texture improved throughout the evaluation period as indicated by the decline in the median value of roughness index (Figure 8). The roughness index was not disparate between the two groups at each follow-up ( $p$-value $>0.05$ ). At the last follow-up, the median roughness index significantly decreased from 18.24 to 16.08 in the RT gel group and from 18.04 to 17.05 in the ALA group.

\section{A}

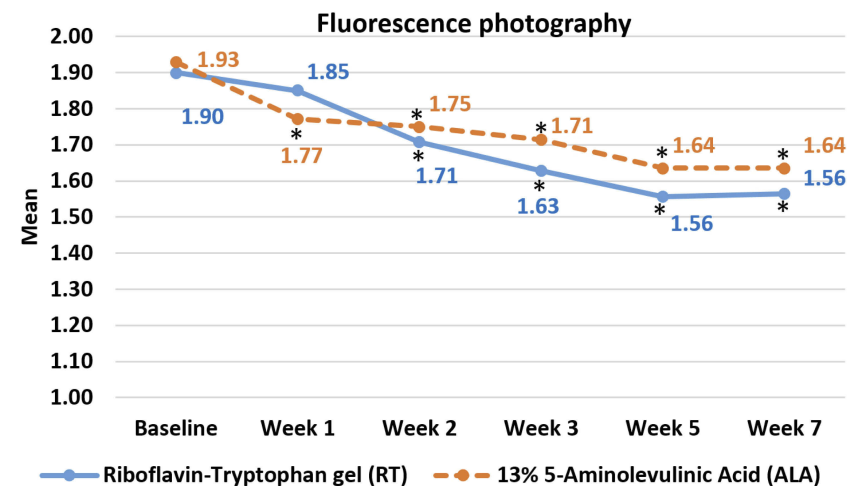

The treatment side-effects were well tolerated in all subjects. Mild erythema patches were significantly recorded in eight subjects treated with PDT on the RT gel facial side and in three subjects treated with PDT on the ALA side $(p<0.05)$. Nevertheless, the patches were gone in only 10-20 mins after the irradiation, with no scarring. A mild burning sensation was reported after applying photosensitizers on the RT gel facial and ALA sides in 18 and 6 subjects, respectively $(p<0.01)$. The burning sensation disappeared after the incubation period ended, and there were no reports of pain, itching, or acne aggravation during the treatment.

\section{Discussion}

Recently, tryptophan and riboflavin were proposed as new photosensitizers for acne treatment. In 2012, Yun ${ }^{18}$ performed a study in which eleven patients with acne vulgaris underwent 4-6 sessions of PDT with tryptophan and riboflavin in 2-3 months. The results showed that PDT with tryptophan and riboflavin could be an effective and safe treatment for acne vulgaris. In this study, we observed a significant reduction in comedone and papule acne counts for PDT with RT gel and with ALA. These results correlate with those of previous studies that reported an acne count reduction with PDT with ALA. ${ }^{5,12}$ PDT may augment epidermal turnover, unplug the follicles, and reduce hyperkeratosis, ${ }^{8}$ and these results would decrease the triggering factors of acne development. Nevertheless, the improvements demonstrated in this study, at the first week after treatment, were observed earlier than those in previous studies. A more significant improvement in comedone acne was seen for PDT with RT gel at week 3.

Natural porphyrins in sebaceous follicles serve as endogenous photosensitizers, which provide advantages after

\section{B}

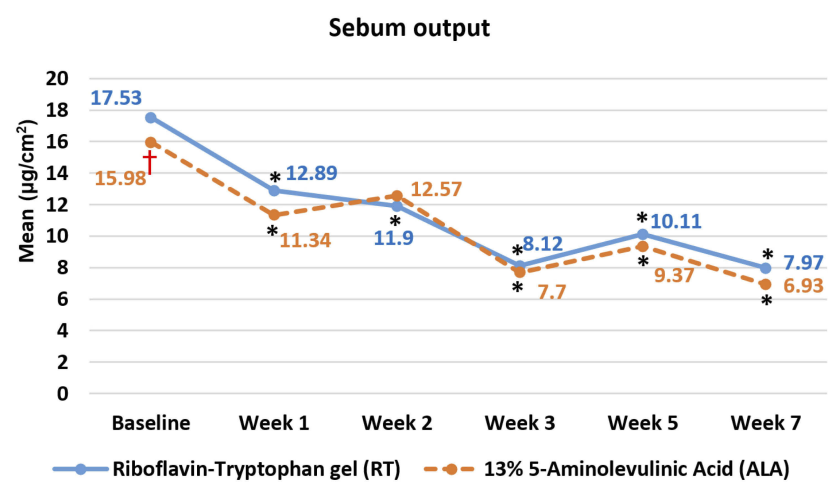

Figure 4 (A) Mean of porphyrin grading throughout the follow-up sessions. (B) Mean of sebum output throughout the follow-up sessions. (*p-value <0.05 comparison to baseline within the therapeutic group, ${ }^{\dagger} \mathrm{p}$-value $<0.05$ for comparison of PDT with RT gel and PDT with ALA). 
A
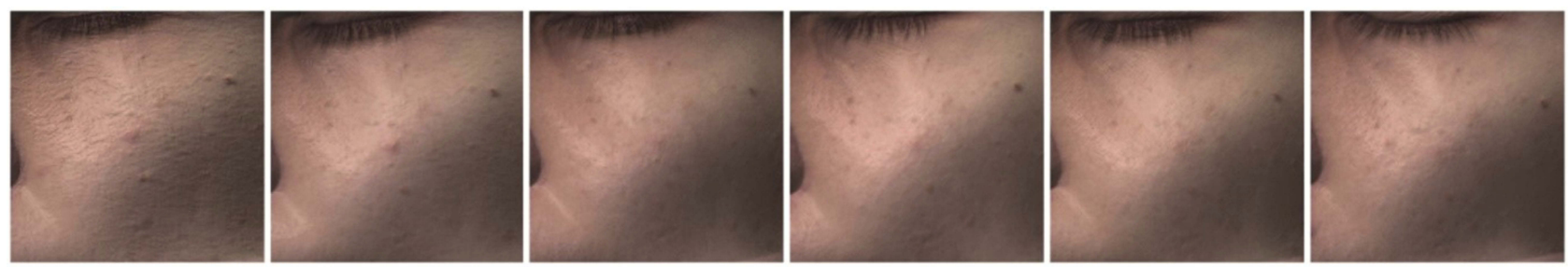

B
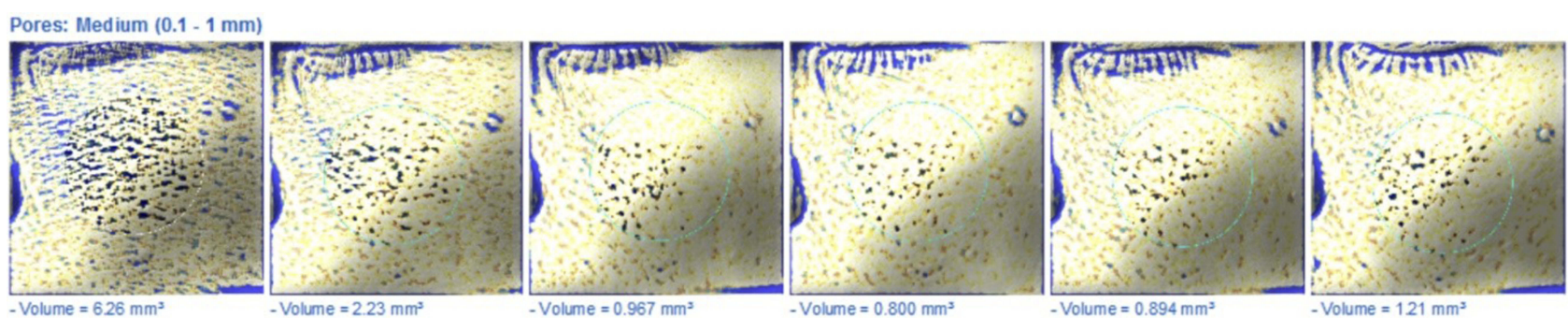

C
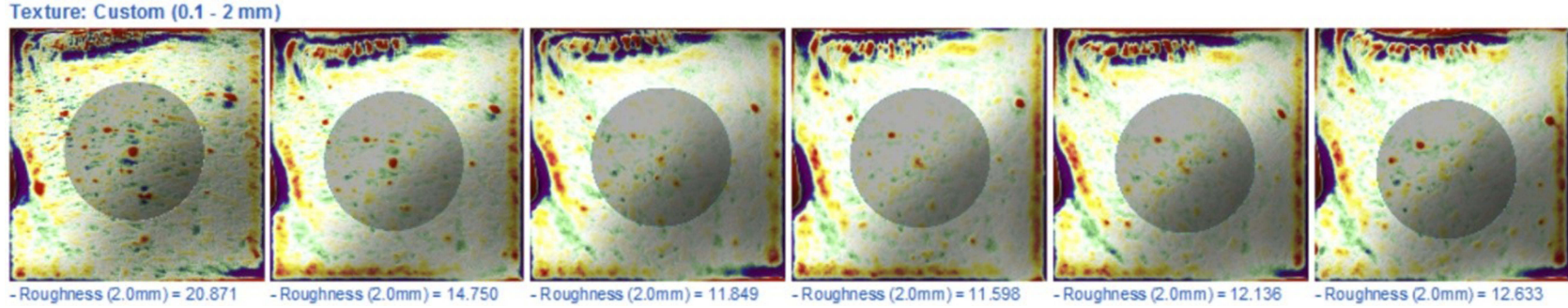

Figure 5 (A) Macroscopic images of the facial skin on the target area of the cheek treated by PDT with RT gel. (B) The volume of the target area of the cheek treated by PDT with RT gel. (C) The roughness index of the target area of the cheek treated by PDT with RT gel, measured by Antera 3D at every evaluation.

exposure to blue or red light sources. ${ }^{19}$ Coproporphyrin III and protoporphyrin IX (PpIX) may be produced by P. acnes and are photosensitizing molecules that are fundamental hemoglobin precursors. Coproporphyrin III can absorb ultraviolet A (UVA) light (320-400 nm) and the blue light spectrum (maximum absorption peak near $415 \mathrm{~nm}$ or the Soret band). UVA and blue light can have antibacterial and antiinflammatory effects. ${ }^{3}$ The anti-inflammatory activity of blue light is attributed to its ability to reduce both the cytokineinduced production of IL-1a and intracellular adhesion molecule-1 (ICAM-1) in keratinocytes. ${ }^{20}$ An in vitro study reported the bactericidal effects of red and blue light with ALA on cultured P. acnes. ${ }^{21}$ Both blue and red LED light can kill $P$. acnes, especially when illumination dose and frequency are increased. However, blue light phototherapy has better efficacy in eradicating $P$. acnes than red light phototherapy. In the current study, the porphyrin level measured by fluorescence photography significantly diminished from the second week in PDT with RT gel and from the first week in PDT with ALA, without any differences between the therapies. Fluorescence photography associated with bacterial Coproporphyrin III and PpIX, which represented P. acne populations. ${ }^{22}$ The outcomes support the mechanism of PDT with RT gel and PDT with ALA that decrease P. acne. Riboflavin with blue light or intense pulsed light activates tryptophan and forms radicals that react with water molecules to generate reactive oxygen species, which decreases $P$. acne and sebum secretions. In 2014, Tim Maisch et $\mathrm{al}^{23}$ performed an in vitro study on the effect of cationic riboflavin derivatives plus visible light for methicillin-resistant Staphylococcus aureus, Enterohemorrhagic, Escherichia coli, Pseudomonas aeruginosa, and Acinetobacter baumannii. The results showed a significant reduction in viable bacteria without human skin cell damage. It was assumed that $P$. acne diminution in PDT with RT gel was also caused by bactericidal effects.

A well-controlled study by Hongcharu et $\mathrm{al}^{7}$ reported a significant acne and sebum output improvement in back acne using PDT with 20\% ALA. The significant improvement began at week 3 after the first treatment and 
A
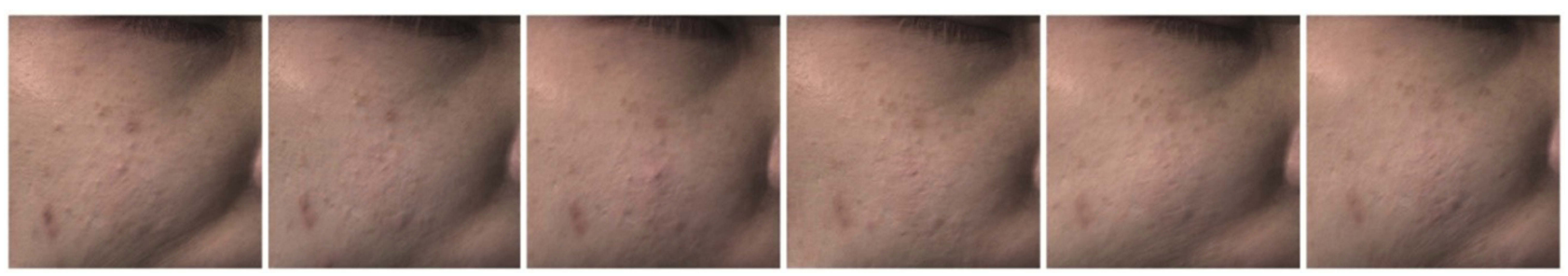

B
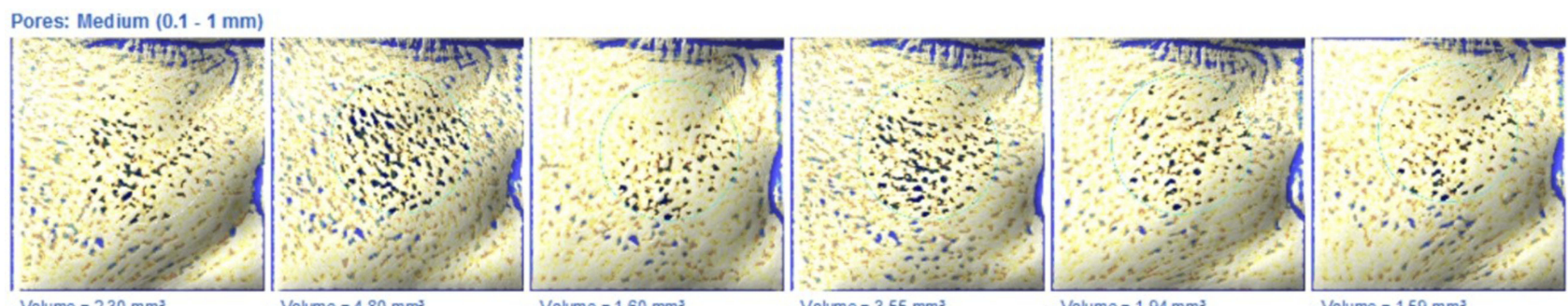

C
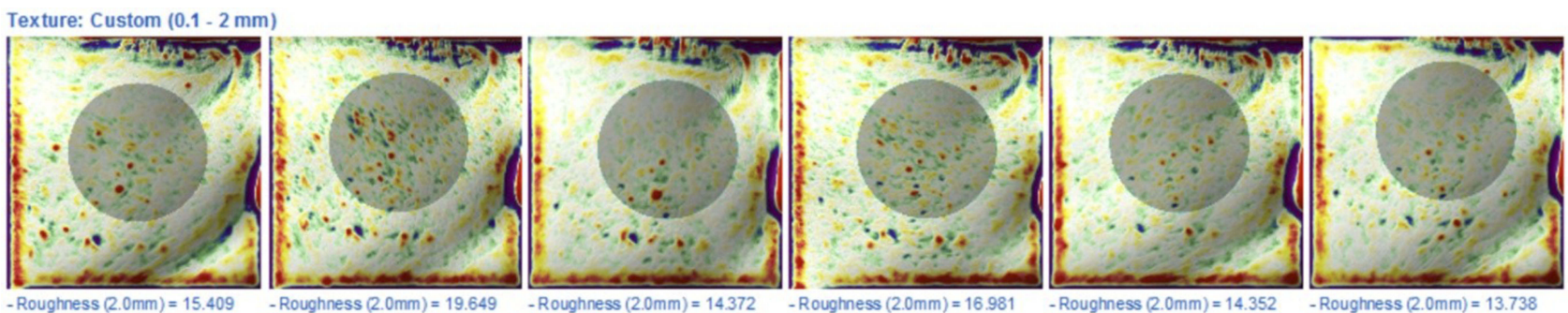

Figure 6 (A) Macroscopic images of the facial skin on the target area of the cheek treated by PDT with ALA. (B) The volume of the target area of the cheek treated by PDT with ALA. (C) The roughness index of the target area of the cheek treated by PDT with ALA, measured by Antera 3D during every evaluation.

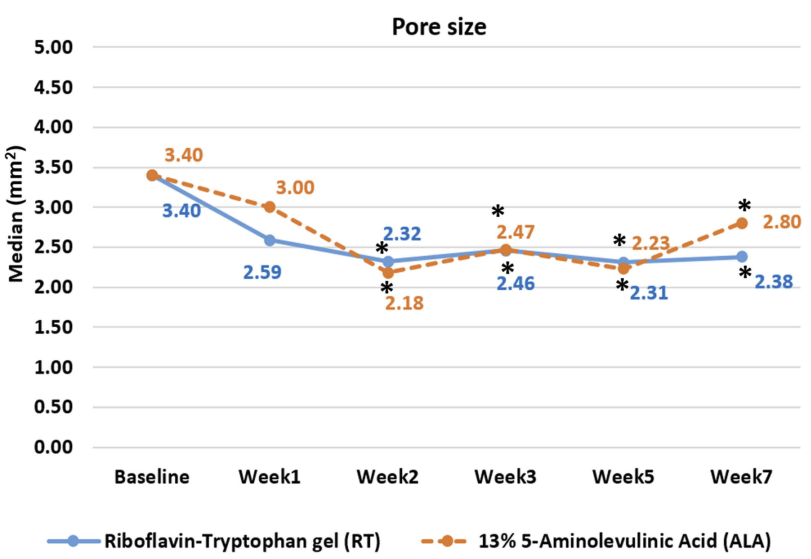

Figure 7 Linear graph illustrating the median value of volume ( ${ }^{*} \mathrm{p}$-value $<0.05$ compared to baseline within the group).

continued for up to 20 weeks of follow-ups. In this study, there was a significant reduction in sebum output within both therapies, with no difference between them. The sebum reduction in this study is explained by the mechanism of direct sebaceous gland damage that led to acne

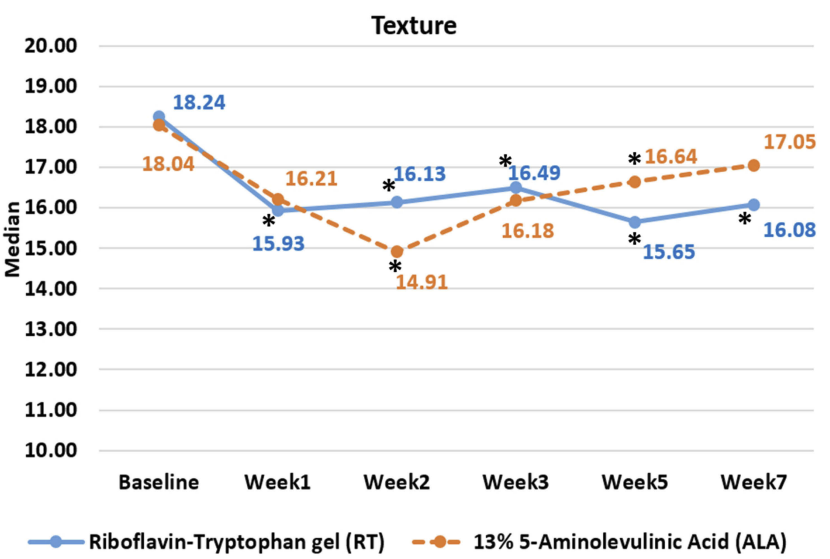

Figure 8 Linear graph illustrating the median value of roughness index (*p-value $<0.05$ compared to baseline within the group).

lesion count reductions. These results confirmed a mode of action for PDT with photosensitizers other than antiinflammatory activity and photodynamic killing of $P$. acnes. We have proposed this PDT mechanism of action, regarding sebaceous gland direct damage, to explain both 
P. acnes and sebum excretion reductions. However, some actions of PDT for acne remain unclear.

The RT gel used in this study also contained minor components such as glycolic acid and niacinamide. Glycolic acid peeling helps reduce comedone, is well tolerated by most skin types, and is a lipid-soluble comedolytic agent that diminishes follicular corneocyte cohesion and aids comedo plug dislodgment. ${ }^{24}$ Glycolic acid also has moderate growth restrictive and bactericidal impacts on P. acnes. ${ }^{25}$ The continuous usage of $2 \%$ topical niacinamide for 4 weeks was shown to significantly decrease sebum excretion in Japanese patients and skin surface sebum levels in Caucasian patients. ${ }^{26}$ $\mathrm{Arca}^{27}$ used a topical 4\% niacinamide gel twice daily and found that it was effective and safe for mild to moderate inflammatory acne. Glycolic acid and niacinamide may have little benefits for acne treatment and may have contributed to the clinical improvements observed in this study.

The results from PDT-ALA for the pore size and skin texture in our clinical trial are similar to those from previous studies in terms of texture improvement. ${ }^{28-30}$ However, in our study, PDT-ALA showed a significant improvement from the second week after treatment onwards, which is earlier than that reported in previous studies. The measured parameters of the pore size and skin texture in this study demonstrated that using RT-gel as a photosensitizer produced non-inferior outcomes compared to ALA. Both PDT with RT-gel and ALA were effective for pore size and skin texture.

Photodynamic therapy plus photosensitizers activate the photochemical biomolecule electron transfer, thus producing reactive oxygen species (ROS). ${ }^{31}$ The activation of ROS or free radicals lead to tissue damage, causing cell apoptosis, mitochondrial damage and activated signaling molecules. Rossi et $\mathrm{al}^{32}$ described the molecular mechanism of rejuvenation that can be achieved by TGF- $\beta$ and FGF production, acceleration of fibroblast activation, and collagen type I-III synthesis. Moreover, PDT decreases the collagen-degrading enzymes, such as matrix metalloproteinases (MMP) 1, 3, and 12. However, the exact molecular mechanism of rejuvenation by PDT on the net dermal collagen production is still not fully understood. A study by Park et $\mathrm{al}^{33}$ summarized that the net MMP levels increased and type I collagen mRNA production decreased, which is in contrast with previous studies. $\mathrm{R}$ Glen Calderhead et $\mathrm{al}^{34}$ proposed that LED phototherapy for skin rejuvenation caused degranulation of the dermal mast cells and induced an inflammatory response, resembling the first phase of wound healing. In 2017, Xiang
Wena ${ }^{35}$ reviewed that PDT elevated the type I and III procollagen production, which led to dermal remodeling. The increase in dermal collagen density and epidermal thickness could improve skin aging.

Although ALA serves as the main effective photosensitizer with PDT for acne treatment, this study demonstrates that RT gel efficacy is as good as ALA efficacy. The findings in both therapies can be explained by antiinflammation effects, direct sebaceous gland damage, and $P$. acnes eradication. Riboflavin-tryptophan may have an inferior side effect profile to ALA in terms of minor side effects. No serious side effects were reported in both groups in this study.

The limitations of this study included the use of an RT gel with small amounts of glycolic and niacinamide, which may improve acne, as well as pore size and texture of the skin. The total study period was 7 weeks, and some additional benefits may have been observed if the follow-up period was longer.

\section{Conclusion}

PDT with RT gel delivers clinical improvement in acne vulgaris non-inferior to PDT with ALA. Both methods demonstrate significant reductions in acne lesion count, acne severity, $P$. acne population, and sebum production. The procedure is non-invasive and effective for the treatment of mild to moderate acne and for reducing pore size and improving skin texture. The side-effects in both therapies were mild, tolerable, and self-resolved within minutes. PDT with RT gel had very good efficacy in treating mild to moderate acne and can be an alternative therapy for acne vulgaris.

\section{Compliance With Ethical Standards}

This manuscript has not been published or presented elsewhere in part or in entirety and is not under consideration by another journal. The study design was approved by the Human Ethics Committee of Thammasat University. We have read and understood your journal's policies, and we believe that neither the manuscript nor the study violates any of these. There are no conflicts of interest to declare.

\section{Acknowledgments}

The authors express sincere gratitude to the financial support provided by Chulabhorn International College of Medicine, Thammasat University, Thailand.

\section{Disclosure}

The authors report no conflicts of interest in this work. 


\section{References}

1. Zaenglein AL, Pathy AL, Schlosser BJ, Alikhan A, Baldwin HE, Berson DS, et al. Guidelines of care for the management of acne vulgaris. J Am Acad Dermatol. 2016;74(5):945-73. e33. doi:10.1016/ j.jaad.2015.12.037

2. Williams HC, Dellavalle RP, Garner S. Acne vulgaris. Lancet. 2012;379(9813):361-372. doi:10.1016/S0140-6736(11)60321-8

3. Sakamoto FH, Lopes JD, Anderson RR. Photodynamic therapy for acne vulgaris: a critical review from basics to clinical practice Part I. Acne vulgaris: when and why consider photodynamic therapy? J Am Acad Dermatol. 2010; 63(2): 183-193. doi:10.1016/j.jaad.2009.09.056

4. Hörfelt C, Funk J, Frohm-Nilsson M, Edström DW, Wennberg AM. Topical methyl aminolaevulinate photodynamic therapy for treatment of facial acne vulgaris: results of a randomized controlled study. $\mathrm{Br} \mathrm{J}$ Dermatol. 2006;155(3):608-613. doi:10.1111/j.1365-2133.2006.07340.x

5. Keyal U, Bhatta AK, Wang XL. Photodynamic therapy for the treatment of different severity of acne: a systematic review. Photodiagnosis Photodyn Ther. 2016;14:191-199. doi:10.1016/j.pdpdt.2016.04.005

6. Barbaric J, Abbott R, Posadzki P, Car M, Gunn L, Layton A, et al. Light therapies for acne: abridged Cochrane systematic review including GRADE assessments. Br J Dermatol. 2018;178(1):61-75. doi:10.1111/bjd.15495

7. Hongcharu W, Taylor C, Aghassi D, Suthamjariya K, Anderson R, Chang Y. Topical ALA-photodynamic therapy for the treatment of acne vulgaris. J Invest Dermatol. 2000;115(2):183-192. doi:10.1046/ j.1523-1747.2000.00046.x

8. Orringer JS, Hammerberg C, Hamilton T, Johnson TM, Kang S, Sachs DL, et al. Molecular effects of photodynamic therapy for photoaging. Arch Dermatol. 2008;144(10):1296-1302. doi:10.1001/ archderm.144.10.1296

9. Hayami J, Okamoto H, Sugihara A, Horio T. Immunosuppressive effects of photodynamic therapy by topical aminolevulinic acid. $J$ Dermatol. 2007;34(5):320-327. doi:10.1111/j.1346-8138.2007.00280.x

10. Friedmann P. Disappearance of epidermal Langerhans cells during PUVA therapy. Br J Dermatol. 1981;105(2):219-221. doi:10.1111/ j.1365-2133.1981.tb01209.x

11. Ma L, Xiang LH, Yu B, Yin R, Chen L, Wu Y, et al. Low-dose topical 5 -aminolevulinic acid photodynamic therapy in the treatment of different severity of acne vulgaris. Photodiagnosis Photodyn Ther. 2013;10(4):583-590. doi:10.1016/j.pdpdt.2013.06.007

12. Sakamoto FH, Torezan L, Anderson RR. Photodynamic therapy for acne vulgaris: a critical review from basics to clinical practice Part II. Understanding parameters for acne treatment with. $\mathrm{J}$ Am Acad Dermatol. 2010;63(2):195-211. doi:10.1016/j.jaad.2009.09.057

13. Common side effects of levulan kerastick (aminolevulinic acid) drug center. RxList; 2016.

14. Casas A, Fukuda H, Batlle AC. Metabolic changes in the heme pathway driven by cyclophosphamide treatment in mice. Cell Mol Biol. 1997;43(1):95-101.

15. Cardoso D, Libardi S, Skibsted L. Riboflavin as a photosensitizer. Effects on human health and food quality. Food Funct. 2012;3(5):487.

16. Buehler BA. Vitamin B2: riboflavin. J Evid Based Complementary Altern Med. 2011;16(2):88-90. doi:10.1177/1533210110392943

17. Faghihi G, Rakhshanpour M, Abtahi-Naeini B, Nilforoushzadeh MA. The efficacy of $5 \%$ dapsone gel plus oral isotretinoin versus oral isotretinoin alone in acne vulgaris: a randomized double-blind study. Adv Biomed Res. 2014;3:177. doi:10.4103/2277-9175.139413
18. Yun YH, Kim TY, Choi IH. Topical photodynamic therapy with triptophan and riboflavin for the treatment of acne vulgaris. $J$ Korean Orient Med Ophthalmol Otolaryngol Dermato. 2012;25 (4):89-98. doi:10.6114/jkood.2012.25.4.089

19. Lee W, Shalita AR, Poh-Fitzpatrick MB. Comparative studies of porphyrin production in propionibacterium acnes and propionibacterium granulosum. $J$ Bacteriol. 1978;133(2):811-815.

20. Shnitkind E, Yaping E, Geen S, Shalita AR, Lee W-L. Anti-inflammatory properties of narrow-band blue light. $J$ Drugs Dermatol. 2006;5(7):605-610.

21. Choi MS, Yun SJ, Beom HJ, Park HR, Lee JB. Comparative study of the bactericidal effects of 5-aminolevulinic acid with blue and red light on propionibacterium acnes. J Dermatol. 2011;38(7):661-666. doi:10.1111/j.1346-8138.2010.01094.x

22. Lucchina LC, Kollias N, Gillies R, et al. Fluorescence photography in the evaluation of acne. J Am Acad Dermatol. 1996;35(1):58-63. doi:10.1016/S0190-9622(96)90497-1

23. Maisch T, Eichner A, Späth A, et al. Fast and effective photodynamic inactivation of multiresistant bacteria by cationic riboflavin derivatives. PLoS One. 2014;9(12):e111792. doi:10.1371/journal.pone.0111792

24. Bolognia J, Jorizzo J, Schaffer J. Acne Vulgaris. Dermatology. 3rd ed. Philadelphia: Elsevier Saunders; 2012:545-559.

25. Takenaka Y, Hayashi N, Takeda M, Ashikaga S, Kawashima M. Glycolic acid chemical peeling improves inflammatory acne eruptions through its inhibitory and bactericidal effects on propionibacterium acnes. J Dermatol. 2012;39(4):350-354. doi:10.1111/j.13468138.2011.01321.x

26. Draelos ZD, Matsubara A, Smiles K. The effect of $2 \%$ niacinamide on facial sebum production. J Cosmet Laser Ther. 2006;8(2):96-101. doi:10.1080/14764170600717704

27. Arca E, Çayırlı M, Koç E, Kurumlu Z. Evaluation of efficacy and safety of niacinamide gel in the treatment of mild to moderate acne. Gulhane Med J. 2013;55:3. doi:10.5455/gulhane.22956

28. Shamban AT. Current and new treatments of photodamaged skin. Facial Plast Surg. 2009;25(5):337-346. doi:10.1055/s-0029-1243083

29. Nootheti PK, Goldman MP. Aminolevulinic acid-photodynamic therapy for photorejuvenation. Dermatol Clin. 2007;25(1):35-45. doi:10.1016/j.det.2006.09.010

30. Goldberg DJ. Photodynamic therapy in skin rejuvenation. Clin Dermatol. 2008;26(6):608-613. doi:10.1016/j.clindermatol.2007.09.009

31. Zhou B, Zhang L, Permatasari F, Liu J, Xu Y, Luo D. ALA-PDT elicits oxidative damage and apoptosis in UVB-induced premature senescence of human skin fibroblasts. Photodiagnosis Photodyn Ther. 2016;14:47-56. doi:10.1016/j.pdpdt.2016.02.005

32. Rossi R, Lotti T, Bruscino N. Photodynamic therapy/assisted photorejuvenation. J Cosmet Dermatol Sci Appl. 2011;1(02):30-35. doi:10.4236/jcdsa.2011.12005

33. Park MY, Sohn S, Lee E-S, Kim YC. Photorejuvenation induced by 5 -aminolevulinic acid photodynamic therapy in patients with actinic keratosis: a histologic analysis. J Am Acad Dermatol. 2010;62(1):8595. doi:10.1016/j.jaad.2009.06.025

34. Calderhead RG, Kubota J, Trelles MA, Ohshiro T. One mechanism behind LED phototherapy for wound healing and skin rejuvenation: key role of the mast cell. Laser Ther. 2008;17(3):141-148. doi:10.5978/islsm.17.141

35. Wen X, Li Y, Hamblin MR. Photodynamic therapy in dermatology beyond non-melanoma cancer: an update. Photodiagnosis Photodyn Ther. 2017;19:140-152. doi:10.1016/j.pdpdt.2017.06.010 


\section{Publish your work in this journal}

Clinical, Cosmetic and Investigational Dermatology is an international, peer-reviewed, open access, online journal that focuses on the latest clinical and experimental research in all aspects of skin disease and cosmetic interventions. This journal is indexed on CAS.
The manuscript management system is completely online and includes a very quick and fair peer-review system, which is all easy to use. Visit http://www.dovepress.com/testimonials.php to read real quotes from published authors. 